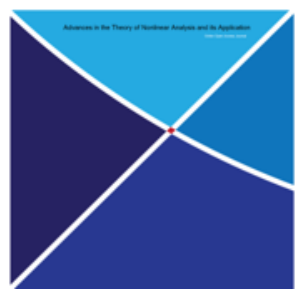

\title{
Existence of Solution to Fractional Order Impulsive Partial Hyperbolic Differential Equations with Infinite Delay
}

\author{
Md. Asaduzzaman ${ }^{\text {a }}$ Md. Zulfikar Ali ${ }^{\text {b }}$ \\ ${ }^{a}$ Department of Mathematics, Islamic University, Kushtia-7003, Bangladesh. \\ ${ }^{b}$ Department of Mathematics, University of Rajshahi, Rajshahi-6205, Bangladesh .
}

\begin{abstract}
In this article, we investigate the existence of solutions to a class of initial value problem ( for short IVP) for fractional order impulsive partial hyperbolic differential equations (for short FOIPHDEs) with infinite delay. Here we use Mixed Riemann-Liouville fractional derivative to construct the considered FOIPHDEs. The analysis of this article is based on Burton-Kirk fixed point theorem. A new existence result for FOIPHDEs with infinite delay has been obtained. To support the analytic proof, we give an illustrative example.
\end{abstract}

Keywords: FOIPHDEs, Burton-Kirk fixed point theorem. 2010 MSC: 26A33, 34K30, 34K37, 35R11.

\section{Introduction}

The purpose of this study is to establish an existence criterion of solutions to the following class of IVP for FOIPHDEs with infinite delay:

$$
\begin{gathered}
\left(D_{z_{k}}^{r} u\right)(x, y)=f\left(x, y, u_{(x, y)}\right) ; \text { if }(x, y) \in J_{k}, k=0,1,2, \cdots, m, \\
u\left(x_{k}^{+}, y\right)=u\left(x_{k}^{-}, y\right)+I_{k}\left(u\left(x_{k}^{-}, y\right)\right) ; i f y \in[0, b], k=1,2, \cdots, m, \\
u(x, y)=\phi(x, y) ; i f(x, y) \in \tilde{J},
\end{gathered}
$$

Email addresses: masad_iu_math@yahoo.com (Md. Asaduzzaman), alimath1964@gmail.com (Md. Zulfikar Ali ) Received November 16, 2019, Accepted: February 28, 2020, Online: March 13, 2020. 


$$
u(x, 0)=\varphi(x), x \in[0, a] \quad u(0, y)=\psi(y), y \in[0, b],
$$

where, $D_{z_{k}}^{r}$ is the mixed Riemann-Liouville fractional differential operator of order $r=\left(r_{1}, r_{2}\right) \in(0,1] \times$ $(0,1], J_{0}=\left[0, x_{1}\right] \times[0, b], J_{k}=\left(x_{k}, x_{k+1}\right] \times[0, b] ; k=1,2, \cdots, m, z_{k}=\left(x_{k}, 0\right) ; k=0,1,2, \cdots, m, a, b>$ $0, J=[0, a] \times[0, b], \tilde{J}=(-\infty, a] \times(-\infty, b] \backslash(0, a] \times(0, b], \varphi:[0, a] \rightarrow \mathbf{R}^{n}, \psi:[0, b] \rightarrow \mathbf{R}^{n}$ are given continuous functions with $\varphi(x)=\phi(x, 0), \psi(y)=\phi(0, y)$ for each $(x, y) \in J, 0=x_{0}<x_{1}<x_{2}<\cdots<$ $x_{m}<x_{m+1}=a, f: J \times B \rightarrow \mathbf{R}^{n}, I_{k}: \mathbf{R}^{n} \rightarrow \mathbf{R}^{n}, k=1,2, \cdots, m, \phi: \tilde{J} \rightarrow \mathbf{R}^{n}$ are given functions and $B$ is a phase space which will be specified in the next section. If $u:(-\infty, a] \times(-\infty, b] \rightarrow \mathbf{R}^{n}$ then for any $(x, y) \in J, u_{(x, y)}$ is defined by

$$
u_{(x, y)}(s, t)=u(x+s, y+t) \text { for }(s, t) \in[-\alpha, 0] \times[-\beta, 0] .
$$

The necessity of fractional order differential equations (for short FDEs) lies in the fact that fractional order model is more accurate than integer order models, that is, there is more degree of freedom in the fractional order models. Furthermore, fractional order derivatives provide an excellent mechanism for the description of memory and hereditary properties of various materials and processes. In applied sense, FDEs arise in various engineering and scientific disciplines for mathematical modeling in the fields of physics, chemistry, biology, fluid flow, electromagnetic theory, polymer rheology, electrical network, statistics, economics, signal and image processing, viscoelasticity, aerodynamics and porous media, etc., see for instance [1, 2, 3, 4, 5, 6, 7, 8, 9, 10, and their cited references. Some recent development of ordinary and partial fractional differential equations can be found in the monographs of Abbas et al. [11, Kilbas et al. [8], Podlubny [10], the papers of Agarwal et al. [12, Asaduzzaman and Zulfikar Ali [13], Zhu et al. [14, Zhang and Fu [15, Agarwal et al. [16], Hemeda [17], Abbas et al. [18, 19], Abbas and Benchohra [20, 21, 22], Agarwal et al. [23], Benchohra et al. [24], Benchohra and Slimani [25], Vityuk and Golushkov [26] and the references therein.

Initial value problems for FOIPHDEs have been addressed by several researchers during last few decades. In current literature, some researchers have been studied the existence of solutions of initial value problems for FOIPHDEs, see for instance [27, 28, 29, 30, 31] and their cited references. Theory of functional differential equations is a significant branch of nonlinear analysis. Functional differential equations or differential delay equations have been used in modeling of different scientific phenomena for long time. Frequently, it has been supposed that the delay is either a fixed constant or is given as an integral in which case it is called a distributed delay, see for instance [32, 33, 34, 35, 36, 37, 38, 39.

On the other hand, theory of impulsive differential equations has become important in some mathematical models of real processes and phenomena studied in physics, chemical technology, population dynamics, biotechnology and economics. There has been a significant development in impulse theory in recent years, especially in the area of impulsive differential equations and inclusions with fixed moments; see for instance [24, 40] and their cited references.

To the best of our knowledge, there is no any work considering the existence of solutions to the initial value problem given by (1) to (4), using Burton-Kirk fixed point theorem [41, 42]. Therefore, our main object is to establish the existence criteria of solutions to the initial value problem for FOIPHDEs given by (1) to (4), using Burton-Kirk fixed point theorem. The rest of this work is furnished as follows: In Section 2, we provide some basic definitions, lemmas and state Burton-Kirk fixed point theorem. Section 3 is used to state and prove our main results, which provide us a technique to check the existence of at least one solutions of initial value problem for FOIPHDEs given by (1) to (4). Finally, in Section 4, we give an example to verify our main result.

\section{Preliminaries Notes}

In this section, we introduce some necessary definitions and preliminary facts which will be used throughout this paper. First, we introduce phase space. The phase space plays an important role in the study of both qualitative and quantitative theory of functional differential equations. To define this space, we usually choose a semi-normed space satisfying some suitable axioms, which was presented by Hale and Kato [38] (see [32, 33, 35]) for functional ordinary differential equations. 
Definition 2.1. (see [32, 33, 35, 38]). For any $(x, y) \in J$, we denote

$$
E_{(x, y)}=[0, x] \times\{0\} \bigcup\{0\} \times[0, y],
$$

furthermore for $x=a, y=b$ we write simply $E$. The phase space $(B,\|.,\|$.$) is a semi-normed linear space$ of functionals mapping from $(-\infty, 0] \times(-\infty, 0]$ into $\boldsymbol{R}^{n}$ satisfying the following fundamental axioms:

(I) If $z:(-\infty, a] \times(-\infty, b] \rightarrow \boldsymbol{R}^{n}$ and $z_{(x, y)} \in B$; for all $(x, y) \in E$, then there are constants $H, K, M>0$ such that for any $(x, y) \in J$, the following conditions hold:

(i) $z_{(x, y)} \in B$,

(ii) $\|z(x, y)\| \leq H\left\|z_{(x, y)}\right\|_{B}$,

(iii) $\left\|z_{(x, y)}\right\|_{B} \leq K \sup _{(s, t) \in[0, x] \times[0, y]}\|z(s, t)\|+M \sup _{(s, t) \in E_{(x, y)}}\left\|z_{(s, t)}\right\|_{B}$,

(II) The space $B$ is complete.

Example 2.2. If the phase space $B$ is a set of all functions $\phi:(-\infty, 0] \times(-\infty, 0] \rightarrow \boldsymbol{R}^{n}$ which are continuous on $[-\alpha, 0] \times[-\beta, 0], \alpha, \beta \geq 0$, with the semi-norm

$$
\|\phi\|_{B}=\sup _{(s, t) \in[-\alpha, 0] \times[-\beta, 0]}\|\phi(s, t)\|,
$$

then we have $H=K=M=1$.

Throughout this paper, $L^{1}\left(J, \mathbf{R}^{n}\right)$ denote the space of Lebesgue-integrable functions $u: J \rightarrow \mathbf{R}^{n}$ with the norm $\|u\|_{L^{1}}=\int_{0}^{a} \int_{0}^{b}\|u(x, y)\| d x d$,$y and C\left(J, \mathbf{R}^{n}\right)$ denote the space of continuous functions $u: J \rightarrow \mathbf{R}^{n}$ with the norm $\|u\|_{\infty}=\sup _{(x, y) \in J}\|u(x, y)\|$.

Among the different definitions of partial fractional derivative and partial fractional integral, the most frequent used definitions are Riemann-Liouville partial fractional integral, Riemann-Liouville partial fractional derivative and Caputo partial fractional derivative, see for instance [2, 8, 10, 11].

Definition 2.3. (see [4, 8, 9]). Let $\alpha \in(0, \infty)$ and $u \in L^{1}\left(J, \boldsymbol{R}^{n}\right)$. The Riemann-Liouville partial fractional integral of order $\alpha$ of $u(x, y)$ with respect to $x$ is defined by

$$
I_{0, x}^{\alpha} u(x, y)=\frac{1}{\Gamma(\alpha)} \int_{0}^{x}(x-s)^{\alpha-1} u(s, y) d s,
$$

for almost all $x \in[0, a]$ and for almost all $y \in[0, b]$, where $\Gamma(\alpha)$ is the Euler Gamma function of $\alpha$ and provided that the integral exists.

Similarly, the Riemann-Liouville partial fractional integral of order $\alpha$ of $u(x, y)$ with respect to $y$ is defined by

$$
I_{0, y}^{\alpha} u(x, y)=\frac{1}{\Gamma(\alpha)} \int_{0}^{y}(y-s)^{\alpha-1} u(x, s) d s,
$$

for almost all $x \in[0, a]$ and for almost all $y \in[0, b]$.

Definition 2.4. (see [4, 8, 9]). Let $\alpha \in(0, \infty)$ and $u \in L^{1}\left(J, \boldsymbol{R}^{n}\right)$. The Riemann-Liouville partial fractional derivative of order $\alpha$ of $u(x, y)$ with respect to $x$ is defined by

$$
\left(D_{0, x}^{\alpha} u\right)(x, y)=\frac{\partial}{\partial x}\left(I_{0, x}^{1-\alpha} u(x, y)\right),
$$

for almost all $x \in[0, a]$ and for almost all $y \in[0, b]$.

Similarly, the Riemann-Liouville partial fractional derivative of order $\alpha$ of $u(x, y)$ with respect to $y$ is defined by

$$
\left(D_{0, y}^{\alpha} u\right)(x, y)=\frac{\partial}{\partial x}\left(I_{0, y}^{1-\alpha} u(x, y)\right),
$$

for almost all $x \in[0, a]$ and for almost all $y \in[0, b]$. 
Definition 2.5. (see [4, 8, 9]). Let $\alpha \in(0, \infty)$ and $u \in L^{1}\left(J, \boldsymbol{R}^{n}\right)$. The Caputo partial fractional derivative of order $\alpha$ of $u(x, y)$ with respect to $x$ is defined by

$$
\left({ }^{C} D_{0, x}^{\alpha} u\right)(x, y)=I_{0, x}^{1-\alpha}\left(\frac{\partial}{\partial x} u(x, y)\right)
$$

for almost all $x \in[0, a]$ and for almost all $y \in[0, b]$.

Similarly, the partial Caputo fractional derivative of order $\alpha$ of $u(x, y)$ with respect to $y$ is defined by

$$
\left({ }^{C} D_{0, y}^{\alpha} u\right)(x, y)=I_{0, y}^{1-\alpha}\left(\frac{\partial}{\partial x} u(x, y)\right),
$$

for almost all $x \in[0, a]$ and for almost all $y \in[0, b]$.

In general, partial Caputo fractional derivative and partial Riemann -Liouville fractional derivative of a function are not same. In particular, the solution space of ${ }^{C} D_{0, y}^{\alpha} u(t)=0$ is spanned by $\left\{1, t, t^{2}, \cdots, t^{n-1}\right\}$, while the solution space of $D_{0, y}^{\alpha} u(t)=0$ is spanned by $\left\{t^{\alpha-1}, t^{\alpha-2}, \cdots, t^{\alpha-n}\right\}$.

Definition 2.6. (see [8, [26]). Let $r=\left(r_{1}, r_{2}\right) \in(0, \infty) \times(0, \infty), z_{k}=\left(x_{k}, 0\right)$ and $u \in L^{1}\left(J, \boldsymbol{R}^{n}\right)$. The left-sided mixed Riemann-Liouville fractional integral of order $r$ of $u(x, y)$ is defined by

$$
\left(I_{z_{k}+}^{r} u\right)(x, y)=\frac{1}{\Gamma\left(r_{1}\right) \Gamma\left(r_{2}\right)} \int_{x_{k}}^{x} \int_{0}^{y}(x-s)^{r_{1}-1}(y-t)^{r_{2}-1} u(s, t) d t d s,
$$

and the right-sided mixed Riemann-Liouville fractional integral of order $r$ of $u(x, y)$ is defined by

$$
\left(I_{z_{k}-}^{r} u\right)(x, y)=\frac{1}{\Gamma\left(r_{1}\right) \Gamma\left(r_{2}\right)} \int_{x}^{x_{k}} \int_{y}^{0}(s-x)^{r_{1}-1}(t-y)^{r_{2}-1} u(s, t) d t d s,
$$

where $\Gamma\left(r_{1}\right) \Gamma\left(r_{2}\right)$ are Euler Gamma function of $r_{1}, r_{2}$ respectively and provided that the integral exists.

Definition 2.7. (see [8, [26]). Let $r=\left(r_{1}, r_{2}\right) \in(0, \infty) \times(0, \infty), z_{k}=\left(x_{k}, 0\right)$ and $u \in L^{1}\left(J, \boldsymbol{R}^{n}\right)$. Then the left-sided mixed Riemann-Liouville fractional derivative of order $r$ of $u$ is defined by

$$
\left(D_{z_{k}+}^{r} u\right)(x, y)=\frac{\partial^{2}}{\partial x \partial y}\left(I_{z_{k}+}^{1-r} u\right)(x, y)
$$

and right-sided mixed Riemann-Liouville fractional derivative of order $r$ of $u$ is defined by

$$
\left(D_{z_{k}-}^{r} u\right)(x, y)=\frac{\partial^{2}}{\partial x \partial y}\left(I_{z_{k}-}^{1-r} u\right)(x, y)
$$

and left-sided mixed Caputo fractional derivative of order $r$ of $u$ is defined by

$$
\left({ }^{C} D_{z_{k}+}^{r} u\right)(x, y)=\left(I_{z_{k}+}^{1-r} \frac{\partial^{2}}{\partial x \partial y} u\right)(x, y)
$$

and right-sided mixed Caputo fractional derivative of order $r$ of $u$ is defined by

$$
\left({ }^{C} D_{z_{k}-}^{r} u\right)(x, y)=\left(I_{z_{k}-}^{1-r} \frac{\partial^{2}}{\partial x \partial y} u\right)(x, y),
$$

where $1-r$ mean that $\left(1-r_{1}, 1-r_{2}\right) \in(0, \infty) \times(0, \infty)$ and $D_{x y}^{2}=\frac{\partial^{2}}{\partial x \partial y}$ denote the mixed second order partial derivative. 
To establish the main result, we need the following generalization of GronwallâĂŹs lemma for two independent variables and singular kernel.

Lemma 2.8. (see [43]). Let $v: J \rightarrow[0, \infty)$ be a real function and $w(.,$.$) be a nonnegative, locally integrable$ function on $J$. If there are constants $c>0$ and $0<r_{1}, r_{2}<1$, such that

$$
v(x, y) \leqslant w(x, y)+c \int_{0}^{x} \int_{0}^{y} \frac{v(s, t)}{(x-s)^{r_{1}}(y-t)^{r_{2}}} d t d s,
$$

then there exists a constant $\delta=\delta\left(r_{1}, r_{2}\right)$ such that

$$
v(x, y) \leqslant w(x, y)+\delta c \int_{0}^{x} \int_{0}^{y} \frac{w(s, t)}{(x-s)^{r_{1}}(y-t)^{r_{2}}} d t d s
$$

for every $(x, y) \in J$.

Theorem 2.9. (Burton-Kirk fixed point theorem) ( see [41, 42]). Let $(X,\|\cdot\|)$ be a Banach space, and $S, T: X \rightarrow X$ two operators satisfying:

(i) $S$ is completely continuous, and

(ii) $T$ is contraction with contraction constant $\alpha<1$.

Then either

(a) the operator equation $u=\lambda T(u / \lambda)+\lambda S(u)$ has a solution in $X$ for $\lambda=1$, or

(b) the set $P=\{u \in X: u=\lambda T(u / \lambda)+\lambda S(u)\}$ of all such solutions is unbounded for $0<\lambda<1$.

Now, we define the solutions of our problem given by (1) to (4) and for these solutions we shall consider the space

$$
\begin{gathered}
\Omega=\left\{u:(-\infty, a] \times(-\infty, b] \rightarrow \mathbf{R}^{n}: u_{(x, y)} \in \text { Bfor }(x, y) \in E\right. \text { and } \\
\exists u\left(x_{k}^{-}, .\right), u\left(x_{k}^{+}, .\right) \text {exist with } u\left(x_{k}^{-}, .\right)=\left(x_{k}, .\right) \\
\left.k=1,2, \cdots, m \text { and } u \in C\left(J_{k}, \mathbf{R}^{n}\right) ; k=0,1, \cdots, m,\right\}
\end{gathered}
$$

where $J_{k}=\left(x_{k}, x_{k+1}\right] \times(0, b]$. Let

$$
J^{\prime}=J \backslash\left\{\left(x_{1}, y\right),\left(x_{2}, y\right), \cdots,\left(x_{m}, y\right), y \in[0, b]\right\} .
$$

For $u \in \Omega$, we define the function $\tilde{u}_{k} \in C\left(\left[x_{k}, x_{k+1}\right] \times[0, b], \mathbf{R}^{n}\right)$ by

$$
\tilde{u}_{k}(x, y)=u(x, y) \text { for }(x, y) \in\left[x_{k}, x_{k+1}\right] \times[0, b]
$$

and

$$
\tilde{u}_{k}\left(x_{k}, y\right)=\lim _{k \rightarrow x_{k}^{+}} u(x, y), \text { for } k=0,1, \cdots, m .
$$

Furthermore, for a set $D \subset \Omega$, we represent the set $\tilde{D}_{k}$ by $\tilde{D}_{k}=\left\{\tilde{u}_{k}: u \in D\right\}$, for $k=0,1, \cdots, m$.

Lemma 2.10. (see [44]). A set $D \subset \Omega$ is relatively compact if and only if, each set $\tilde{D}_{k}$, for $k=0,1, \cdots, m$, is relatively compact in $C\left(\left[x_{k}, x_{k+1}\right] \times[0, b], \boldsymbol{R}^{n}\right)$.

Definition 2.11. A function $u \in \Omega$ is said to a solution of the IVP given by (1) to (4) if u satisfies $\left(D_{0}^{r}(x, y)\right)=f(x, y, u(x)$,$) on J^{\prime}$.

Let us consider $h \in C\left(\left[x_{k}, x_{k+1}\right] \times[0, b], \mathbf{R}^{n}\right), z_{k}=\left(x_{k}, 0\right)$ and

$$
\mu_{k}(x, y)=u(x, 0)+u\left(x_{k}^{+}, y\right)-u\left(x_{k}^{+}, 0\right), k=0,1, \cdots, m .
$$

The following lemma will be needed to establish the existence of solutions of our problem given by (1) to (4): 
Lemma 2.12. A function $u \in C\left(\left[x_{k}, x_{k+1}\right] \times[0, b], \boldsymbol{R}^{n}\right), k=0,1, \cdots, m$ is a solution of following partial fractional differential equation

$$
\left(D_{z_{k}}^{r} u\right)(x, y)=h(x, y) ;(x, y) \in\left[x_{k}, x_{k+1}\right] \times[0, b],
$$

if and only if $u(x, y)$ satisfies

$$
u(x, y)=\mu_{k}(x, y)+\left(I_{z_{k}}^{r} h\right)(x, y) ;(x, y) \in\left[x_{k}, x_{k+1}\right] \times[0, b] .
$$

Proof. Let $u(x, y)$ be a solution of $\left(D_{z_{k}}^{r} u\right)(x, y)=h(x, y) ;(x, y) \in\left[x_{k}, x_{k+1}\right] \times[0, b]$. Then from the definition of the derivative $\left(D_{z_{k}}^{r} u\right)(x, y)$, we have

$$
\frac{\partial^{2}}{\partial x \partial y}\left(I_{z_{k}}^{1-r} u\right)(x, y)=h(x, y)
$$

Hence, we obtain that

$$
I_{z_{k}}^{r}\left(\frac{\partial^{2}}{\partial x \partial y}\left(I_{z_{k}}^{1-r} u\right)\right)(x, y)=\left(I_{z_{k}}^{r} h\right)(x, y)
$$

which gives us

$$
I_{z_{k}}^{1}\left(\frac{\partial^{2}}{\partial x \partial y} u\right)(x, y)=\left(I_{z_{k}}^{r} h\right)(x, y)
$$

But we know that

$$
I_{z_{k}}^{1}\left(\frac{\partial^{2}}{\partial x \partial y} u\right)(x, y)=u(x, y)-u(x, 0)-u\left(x_{k}^{+}, y\right)+u\left(x_{k}^{+}, 0\right) .
$$

So from (20), we yield that

$$
u(x, y)=\mu_{k}(x, y)+\left(I_{z_{k}}^{r} h\right)(x, y), \forall(x, y) \in\left[x_{k}, x_{k+1}\right] \times[0, b] .
$$

Conversely, suppose that $u(x, y)$ satisfy (17). Then, it is obvious that $u(x, y)$ must be a solution of $\left(D_{z_{k}}^{r} u\right)(x, y)=h(x, y)$, for all $(x, y) \in\left[x_{k}, x_{k+1}\right] \times[0, b]$. This completes the proof.

Lemma 2.13. Let $0<r_{1}, r_{2} \leq 1$ and let $h: J \rightarrow \boldsymbol{R}^{n}$ be a continuous function. Then the function $u(x, y)$ is a solution of the fractional integral equation

$$
u(x, y)=\left\{\begin{array}{lc}
\phi(x, y) & \text { if }(x, y) \in \tilde{J}, \\
\mu(x, y)+\sum_{0<x_{k}<x}\left(I_{k}\left(u\left(x_{k}^{-}, y\right)\right)-I_{k}\left(u\left(x_{k}^{-}, 0\right)\right)\right) & \text { if }(x, y) \in J \\
+\frac{1}{\Gamma\left(r_{1}\right) \Gamma\left(r_{2}\right)} \sum_{0<x_{k}<x} \int_{x_{k-1}}^{x_{k}} \int_{0}^{y}\left(x_{k}-s\right)^{r_{1}-1}(y-t)^{r_{2}-1} h(s, t) d t d s \\
+\frac{1}{\Gamma\left(r_{1}\right) \Gamma\left(r_{2}\right)} \int_{x_{k}}^{x} \int_{0}^{y}(x-s)^{r_{1}-1}(y-t)^{r_{2}-1} h(s, t) d t d s, k=1,2, \cdots, m
\end{array}\right.
$$

if and only if $u(x, y)$ is a solution of the fractional IVP

$$
\begin{aligned}
& \left(D_{z_{k}}^{r} u\right)(x, y)=h(x, y) ;(x, y) \in J_{k}, k=0,1, \cdots, m, \\
& u\left(x_{k}^{+}, y\right)=u\left(x_{k}^{-}, y\right)+I_{k} u\left(x_{k}^{-}, y\right), k=1,2, \cdots, m .
\end{aligned}
$$

Proof. The proof of this lemma follows from the Lemma 2.12 . 


\section{Main Results}

This section is devoted to establish the existence criteria for solutions to a class of initial value problem for fractional order impulsive partial hyperbolic differential equations with infinite delay given by (1) to (4) applying a fixed point theorem (Theorem 2.9) due to Burton and Kirk [41, 42]. To establish the desired existence criteria, we need the following assumptions:

$\left(A_{1}\right)$ The functions $I_{k}: \mathbf{R}^{n} \rightarrow \mathbf{R}^{n}$ and $f: J \times B \rightarrow \mathbf{R}^{n}$ are continuous.

$\left(A_{2}\right)$ There exists $p, q \in C\left(J, \mathbf{R}_{+}\right)$such that

$$
\|f(t, x, u)\| \leq p(t, x)+q(t, x)\|u\|_{B} \text { for }(t, x) \in J \text { and } u \in B .
$$

$\left(A_{3}\right)$ There exists $l>0$ such that

$$
\left\|I_{k}(u)-I_{k}(v)\right\| \leq l\|u-v\| \text { for each } u, v \in \mathbf{R}^{n} .
$$

We are now in position to present and prove our main results.

Theorem 3.1. Suppose that the assumptions $\left(A_{1}\right),\left(A_{2}\right)$ and $\left(A_{3}\right)$ are hold. If there exists $l>0$ such that

$$
2 m l<1
$$

then the initial value problem given by (1) to (4) has least one solution on $J$.

Proof. We shall diminish the existence of solutions of IVP given by (1) to (4) to a fixed point problem. Consider an operator $A: \Omega \rightarrow \Omega$ which is defined as follows:

$$
A(u)(x, y)= \begin{cases}\phi(x, y) & \text { if }(x, y) \in \tilde{J}, \\ \mu(x, y)+\sum_{0<x_{k}<x}\left(I_{k}\left(u\left(x_{k}^{-}, y\right)\right)-I_{k}\left(u\left(x_{k}^{-}, 0\right)\right)\right) & \text { if }(x, y) \in J, \\ +\frac{1}{\Gamma\left(r_{1}\right) \Gamma\left(r_{2}\right)} \sum_{0<x_{k}<x} \int_{x_{k-1}}^{x_{k}} \int_{0}^{y}\left(x_{k}-s\right)^{r_{1}-1}(y-t)^{r_{2}-1} h(s, t) d t d s \\ +\frac{1}{\Gamma\left(r_{1}\right) \Gamma\left(r_{2}\right)} \int_{x_{k}}^{x} \int_{0}^{y}(x-s)^{r_{1}-1}(y-t)^{r_{2}-1} h(s, t) d t d s, k=1,2, \cdots, m,\end{cases}
$$

Now, we set two operators $S, T: \Omega \rightarrow \Omega$, which are defined in the following way:

$$
S(u)(x, y)=\left\{\begin{array}{lr}
\phi(x, y) & \text { if }(x, y) \in \tilde{J}, \\
\frac{1}{\Gamma\left(r_{1}\right) \Gamma\left(r_{2}\right)} \sum_{0<x_{k}<x} \int_{x_{k-1}}^{x_{k}} \int_{0}^{y}\left(x_{k}-s\right)^{r_{1}-1}(y-t)^{r_{2}-1} & \text { if }(x, y) \in J, \\
f(s, t, u(s, t)) d t d s+\frac{1}{\Gamma\left(r_{1}\right) \Gamma\left(r_{2}\right)} \int_{x_{k}}^{x} \int_{0}^{y}(x-s)^{r_{1}-1}(y-t)^{r_{2}-1} & k=1,2, \cdots, m \\
f(s, t, u(s, t)) d t d s, & k, m
\end{array}\right.
$$

and

$$
T(u)(x, y)= \begin{cases}0, & \text { if }(x, y) \in \tilde{J} \\ \mu(x, y) & \text { if }(x, y) \in J \\ +\sum_{0<x_{k}<x}\left(I_{k}\left(u\left(x_{k}^{-}, y\right)\right)-I_{k}\left(u\left(x_{k}^{-}, 0\right)\right)\right), & k=1,2, \cdots, m .\end{cases}
$$

Let $v:(-\infty, a] \times(-\infty, b] \rightarrow \mathbf{R}^{n}$ be a function defined by

$$
v(x, y)= \begin{cases}\phi(x, y), & \text { if }(x, y) \in \tilde{J} \\ \mu(x, y), & \text { if }(x, y) \in J\end{cases}
$$

Then $v_{(x, y)}=\phi$ for all $(x, y) \in E$.

Now for each $w \in\left(J, \mathbf{R}^{n}\right)$ with $w(x, y)=0$ for every $(x, y) \in E$, we define a function $\bar{w}$ by

$$
\bar{w}(x, y)= \begin{cases}0, & \text { if }(x, y) \in \tilde{J}, \\ w(x, y), & \text { if }(x, y) \in J .\end{cases}
$$


If $u$ satisfies the integral equation,

$$
u(x, y)=\mu(x, y)+\frac{1}{\Gamma\left(r_{1}\right) \Gamma\left(r_{2}\right)} \int_{0}^{x} \int_{0}^{y}(x-s)^{r_{1}-1}(y-t)^{r_{2}-1} f\left(s, t, u_{(s, t)}\right) d t d s,
$$

then we can decompose $u$ as $u(x, y)=\bar{w}(x, y)+v(x, y) ;(x, y) \in\left(x_{k}, x_{k+1}\right] \times[0, b]$, which implies that $u_{(x, y)}=\bar{w}_{(x, y)}+v_{(x, y)}$, for every $(x, y) \in J \times[0, b]$, and the function $w$ satisfies

$$
\begin{aligned}
w(x, y) & =\sum_{0<x_{k}<x}\left(I_{k}\left(u\left(x_{k}^{-}, y\right)\right)-I_{k}\left(u\left(x_{k}^{-}, 0\right)\right)\right)+\frac{1}{\Gamma\left(r_{1}\right) \Gamma\left(r_{2}\right)} \\
& \cdot \sum_{0<x_{k}<x} \int_{x_{k-1}}^{x_{k}} \int_{0}^{y}\left(x_{k}-s\right)^{r_{1}-1}(y-t)^{r_{2}-1} f\left(s, t, \bar{w}_{(s, t)}+v_{(s, t)}\right) d t d s \\
& +\frac{1}{\Gamma\left(r_{1}\right) \Gamma\left(r_{2}\right)} \int_{x_{k}}^{x} \int_{0}^{y}(x-s)^{r_{1}-1}(y-t)^{r_{2}-1} f\left(s, t, \bar{w}_{(s, t)}+v_{(s, t)}\right) d t d s .
\end{aligned}
$$

Now, if we set

$$
B_{0}=\{w \in \Omega: w(x, y)=0 \text { for }(x, y) \in E\},
$$

and let $\|\cdot\|_{B_{0}}$ be the norm in $B_{0}$, which is defined by

$$
\|w\|_{B_{0}}=\sup _{(x, y) \in E}\left\|w_{(x, y)}\right\|_{B}+\sup _{(x, y) \in J}\|w(x, y)\|=\sup _{(x, y) \in J}\|w(x, y)\|, w \in B_{0}
$$

then it is clear that $B_{0}$ is a Banach space with norm $\|\cdot\|_{B_{0}}$. If we consider two operators $S, T: B_{0} \rightarrow B_{0}$, which are defined in the following way:

$$
S(w)(x, y)=\left\{\begin{array}{l}
\frac{1}{\Gamma\left(r_{1}\right) \Gamma\left(r_{2}\right)} \sum_{0<x_{k}<x} \int_{x_{k-1}}^{x_{k}} \int_{0}^{y}\left(x_{k}-s\right)^{r_{1}-1}(y-t)^{r_{2}-1} \\
f\left(s, t, \bar{w}_{(s, t)}+v_{(s, t)}\right) d t d s+\frac{1}{\Gamma\left(r_{1}\right) \Gamma\left(r_{2}\right)} \int_{x_{k}}^{x} \int_{0}^{y}(x-s)^{r_{1}-1} \\
(y-t)^{r_{2}-1} f\left(s, t, \bar{w}_{(s, t)}+v_{(s, t)}\right) d t d s \\
\text { where }(x, y) \in J, k=1,2, \cdots, m
\end{array}\right.
$$

and

$$
T(w)(x, y)=\mu(x, y)+\sum_{0<x_{k}<x}\left(I_{k}\left(u\left(x_{k}^{-}, y\right)\right)-I_{k}\left(u\left(x_{k}^{-}, 0\right)\right)\right)
$$

where $(x, y) \in J, k=1,2, \cdots, m$,

then the problem of finding solutions of the IVP given by (1) to (4) is diminished to finding solutions of the operator equation $S(w)+T(w)=w$. To prove this theorem, we shall prove that the operators $S$ and $T$ satisfy all the conditions of Theorem 2.9. The proof will be completed in the following steps.

Step-1: In this step we prove that the operator $S$ is continuous.

Let $\left\{w_{n}\right\}$ be a sequence such that $w_{n} \rightarrow w$ as $n \rightarrow \infty$ in $B_{0}$, then for each $(x, y) \in J$ we have

$$
\begin{aligned}
& \left\|S\left(w_{n}\right)(x, y)-S(w)(x, y)\right\| \\
& \leq \frac{1}{\Gamma\left(r_{1}\right) \Gamma\left(r_{2}\right)} \sum_{0<x_{k}<x} \int_{x_{k-1}}^{x_{k}} \int_{0}^{y}\left(x_{k}-s\right)^{r_{1}-1}(y-t)^{r_{2}-1} \\
& \left\|f\left(s, t, \bar{w}_{n(s, t)}+v_{n(s, t)}\right)-f\left(s, t, \bar{w}_{(s, t)}+v_{(s, t)}\right)\right\| d t d s+\frac{1}{\Gamma\left(r_{1}\right) \Gamma\left(r_{2}\right)} \int_{x_{k}}^{x} \int_{0}^{y} \\
& (x-s)^{r_{1}-1}(y-t)^{r_{2}-1} \mid f\left(s, t, \bar{w}_{n(s, t)}+v_{n(s, t)}\right)-f\left(s, t, \bar{w}_{(s, t)}+v_{(s, t)}\right) \| d t d s \\
& \leq \frac{\left\|f\left(., . ., \bar{w}_{n(. . .)}+v_{n(. . .)}\right)-f\left(., ., \bar{w}_{(. . .)}+v_{(., .)}\right)\right\|}{\Gamma\left(r_{1}\right) \Gamma\left(r_{2}\right)}\left[\sum _ { 0 < x _ { k } < x } \left(\int_{x_{k-1}}^{x_{k}} \int_{0}^{y}\right.\right. \\
& \left.\left.\left(x_{k}-s\right)^{r_{1}-1}(y-t)^{r_{2}-1} d t d s\right)+\int_{x_{k}}^{x} \int_{0}^{y}(x-s)^{r_{1}-1}(y-t)^{r_{2}-1} d t d s\right] .
\end{aligned}
$$


Since, $f$ is continuous function, then we have

$$
\left\|S\left(w_{n}\right)-S(w)\right\|_{B_{0}} \leq \frac{2 a^{r_{1}} b^{r_{2}}\left\|f\left(., ., \bar{w}_{n(., .)}\right)-f\left(., ., \bar{w}_{(., .)}\right)\right\|_{\infty}}{\Gamma\left(r_{1}+1\right) \Gamma\left(r_{2}+1\right)} \rightarrow 0, \text { as } n \rightarrow \infty .
$$

Therefore, the operator $S$ is continuous.

Step-2: In this step we prove that the operator $S$ maps on bounded sets in $B_{0}$.

To complete this step, it is sufficient to prove that for any $m^{*}$, there exists a positive constant $\tau$ such that, $\|S(w)\|_{B_{0}} \leq \tau$ for every $w \in B_{m^{*}}=\left\{w \in B_{0}:\|w\|_{B_{0}} \leq m^{*}\right\}$.

From assumption $\left(A_{2}\right)$ we have for each $(x, y) \in\left(x_{k}, x_{k+1}\right] \times[0, b]$,

$$
\begin{aligned}
& \|S(w)(x, y)\| \\
& \leq \frac{1}{\Gamma\left(r_{1}\right) \Gamma\left(r_{2}\right)} \sum_{k=1}^{m} \int_{x_{k-1}}^{x_{k}} \int_{0}^{y}\left(x_{k}-s\right)^{r_{1}-1}(y-t)^{r_{2}-1}\left\|f\left(s, t, \bar{w}_{(s, t)}+v_{(s, t)}\right)\right\| d t d s \\
& +\frac{1}{\Gamma\left(r_{1}\right) \Gamma\left(r_{2}\right)} \int_{x_{k}}^{x} \int_{0}^{y}(x-s)^{r_{1}-1}(y-t)^{r_{2}-1}\left\|f\left(s, t, \bar{w}_{(s, t)}+v_{(s, t)}\right)\right\| d t d s \\
& \leq \frac{\|p\|_{\infty}+\|q\|_{\infty} m^{*}}{\Gamma\left(r_{1}\right) \Gamma\left(r_{2}\right)}\left[\sum_{k=1}^{m}\left(\int_{x_{k-1}}^{x_{k}} \int_{0}^{y}\left(x_{k}-s\right)^{r_{1}-1}(y-t)^{r_{2}-1} d t d s\right)\right. \\
& \left.+\int_{x_{k}}^{x} \int_{0}^{y}(x-s)^{r_{1}-1}(y-t)^{r_{2}-1} d t d s\right] .
\end{aligned}
$$

Hence

$$
\|S(w)\|_{B} \leq \frac{2 a^{r_{1}} b^{r_{2}}\left(\|p\|_{\infty}+\|q\|_{\infty} m^{*}\right)}{\Gamma\left(r_{1}+1\right) \Gamma\left(r_{2}+1\right)}=\tau(\text { say })
$$

where

$$
\left\|\bar{w}_{(s, t)}+v_{(s, t)}\right\|_{B} \leq\left\|\bar{w}_{(s, t)}\right\|_{B}+\left\|v_{(s, t)}\right\|_{B} \leq K m^{*}+K\|\phi(0,0)\|+M\|\phi\|_{B}=\xi(s a y) .
$$

Therefore,

$$
\|S(w)\|_{B_{0}} \leq \tau
$$

Step-3: In this step we prove that the operator $S$ maps from bounded sets into equicontinuous in $B_{0}$. Let $\left(x_{1}, y_{1}\right),\left(x_{2}, y_{2}\right) \in(0, a] \times(0, b], x_{1}<x_{2}, y_{1}<y_{2}$ and $B_{m^{*}}$ be a bounded set as in the step-2. Now for any $w \in B_{m^{*}}$, we have

$$
\begin{aligned}
& \left\|S(w)\left(x_{2}, y_{2}\right)-S(w)\left(x_{1}, y_{1}\right)\right\| \\
& \leq \frac{1}{\Gamma\left(r_{1}\right) \Gamma\left(r_{2}\right)} \sum_{k=1}^{m} \int_{x_{k-1}}^{x_{k}} \int_{0}^{y_{1}}\left(x_{k}-s\right)^{r_{1}-1}\left[\left(y_{2}-t\right)^{r_{2}-1}-\left(y_{1}-t\right)^{r_{2}-1}\right] \\
& f\left(s, t, \bar{w}_{(s, t)}+v_{(s, t)}\right) d t d s+\frac{1}{\Gamma\left(r_{1}\right) \Gamma\left(r_{2}\right)} \sum_{k=1}^{m} \int_{x_{k-1}}^{x_{k}} \int_{y_{1}}^{y_{2}}\left(x_{k}-s\right)^{r_{1}-1}\left(y_{2}-t\right)^{r_{2}-1} \\
& \left\|f\left(s, t, \bar{w}_{(s, t)}+v_{(s, t)}\right)\right\| d t d s+\frac{1}{\Gamma\left(r_{1}\right) \Gamma\left(r_{2}\right)} \int_{0}^{x_{1}} \int_{0}^{y_{1}}\left[\left(x_{2}-s\right)^{r_{1}-1}\left(y_{2}-t\right)^{r_{2}-1}\right. \\
& \left.-\left(x_{1}-s\right)^{r_{1}-1}\left(y_{1}-t\right)^{r_{2}-1}\right] f\left(s, t, \bar{w}_{(s, t)}+v_{(s, t)}\right) d t d s \\
& +\frac{1}{\Gamma\left(r_{1}\right) \Gamma\left(r_{2}\right)} \int_{x_{1}}^{x_{2}} \int_{y_{1}}^{y_{2}}\left(x_{2}-s\right)^{r_{1}-1}\left(y_{2}-t\right)^{r_{2}-1}\left\|f\left(s, t, \bar{w}_{(s, t)}+v_{(s, t)}\right)\right\| d t d s \\
& +\frac{1}{\Gamma\left(r_{1}\right) \Gamma\left(r_{2}\right)} \int_{0}^{x_{1}} \int_{y_{1}}^{y_{2}}\left(x_{2}-s\right)^{r_{1}-1}\left(y_{2}-t\right)^{r_{2}-1}\left\|f\left(s, t, \bar{w}_{(s, t)}+v_{(s, t)}\right)\right\| d t d s
\end{aligned}
$$




$$
\begin{aligned}
& +\frac{1}{\Gamma\left(r_{1}\right) \Gamma\left(r_{2}\right)} \int_{x_{1}}^{x_{2}} \int_{0}^{y_{1}}\left(x_{2}-s\right)^{r_{1}-1}\left(y_{2}-t\right)^{r_{2}-1}\left\|f\left(s, t, \bar{w}_{(s, t)}+v_{(s, t)}\right)\right\| d t d s \\
& \leq \frac{\|p\|_{\infty}+\|q\|_{\infty} m^{*}}{\Gamma\left(r_{1}+1\right) \Gamma\left(r_{2}+1\right)}\left[\sum _ { k = 1 } ^ { m } \left(\int _ { x _ { k - 1 } } ^ { x _ { k } } \int _ { 0 } ^ { y _ { 1 } } ( x _ { k } - s ) ^ { r _ { 1 } - 1 } \left[\left(y_{2}-t\right)^{r_{2}-1}\right.\right.\right. \\
& \left.\left.\quad-\left(y_{1}-t\right)^{r_{2}-1}\right] d t d s\right)+\sum_{k=1}^{m}\left(\int_{x_{k-1}}^{x_{k}} \int_{y_{1}}^{y_{2}}\left(x_{k}-s\right)^{r_{1}-1}\left(y_{2}-t\right)^{r_{2}-1} d t d s\right) \\
& \quad+\int_{0}^{x_{1}} \int_{0}^{y_{1}}\left[\left(x_{2}-s\right)^{r_{1}-1}\left(y_{2}-t\right)^{r_{2}-1}-\left(x_{1}-s\right)^{r_{1}-1}\left(y_{1}-t\right)^{r_{2}-1}\right] d t d s \\
& \quad+\int_{x_{1}}^{x_{2}} \int_{y_{1}}^{y_{2}}\left(x_{2}-s\right)^{r_{1}-1}\left(y_{2}-t\right)^{r_{2}-1} d t d s \\
& \quad+\int_{0}^{x_{1}} \int_{y_{1}}^{y_{2}}\left(x_{2}-s\right)^{r_{1}-1}\left(y_{2}-t\right)^{r_{2}-1} d t d s \\
& \left.\quad+\int_{x_{1}}^{x_{2}} \int_{0}^{y_{1}}\left(x_{2}-s\right)^{r_{1}-1}\left(y_{2}-t\right)^{r_{2}-1} d t d s\right] \\
& \| \frac{\|p\|_{\infty}+\|q\|_{\infty} m^{*}}{\Gamma\left(r_{1}+1\right) \Gamma\left(r_{2}+1\right)}\left[\sum _ { k = 1 } ^ { m } \left(\int _ { x _ { k - 1 } } ^ { x _ { k } } \int _ { 0 } ^ { y _ { 1 } } ( x _ { k } - s ) ^ { r _ { 1 } - 1 } \left[\left(y_{2}-t\right)^{r_{2}-1}\right.\right.\right. \\
& \left.\left.-\left(y_{1}-t\right)^{r_{2}-1}\right] d t d s\right)+\sum_{k=1}^{m}\left(\int_{x_{k-1}}^{x_{k}} \int_{y_{1}}^{y_{2}}\left(x_{k}-s\right)^{r_{1}-1}\left(y_{2}-t\right)^{r_{2}-1} d t d s\right) \\
& \left.+\left(2 y_{2}^{r_{2}}\left(x_{2}-x_{1}\right)^{r_{1}}+2 x_{2}^{r_{1}}\left(y_{2}-y_{1}\right)^{r_{2}}+x_{1}^{r_{1}} y_{1}^{r_{2}}-x_{2}^{r_{1}} y_{2}^{r_{2}}-2\left(x_{2}-x_{1}\right)^{r_{1}}\left(y_{2}-y_{1}\right)^{r_{2}}\right)\right] \\
& \rightarrow 0 a s x_{1} \rightarrow x_{2} \text { and } y_{1} \rightarrow y_{2} .
\end{aligned}
$$

Hence, the operator $S$ maps from bounded sets into equicontinuous in $B_{0}$.

Combining the consequences of step-1 to step-3 and applying the Arzela-Ascoli theorem [45], we can accomplish that the operator $S: B_{0} \rightarrow B_{0}$ is completely continuous.

Step-4: In this step we prove that the operator $T$ is contraction. Let $w, w^{*} \in B_{0}$, then for each $(x, y) \in J$ we have

$$
\begin{aligned}
& \left\|T(w)(x, y)-T\left(w^{*}\right)(x, y)\right\| \\
& \leq \sum_{k=1}^{m}\left(\left\|I_{k}\left(w\left(x_{k}^{-}, y\right)\right)-I_{k}\left(w^{*}\left(x_{k}^{-}, y\right)\right)\right\|+\left\|I_{k}\left(w\left(x_{k}^{-}, 0\right)\right)-I_{k}\left(w^{*}\left(x_{k}^{-}, 0\right)\right)\right\|\right) \\
& \leq \sum_{k=1}^{m} l\left(\left\|w-w^{*}\right\|_{B_{0}}+\left\|w-w^{*}\right\|_{B_{0}}\right) \leq 2 m l\left\|w-w^{*}\right\|_{B_{0}} .
\end{aligned}
$$

Thus,

$$
\left\|T(w)-T\left(w^{*}\right)\right\| \leq 2 m l\left\|w-w^{*}\right\|_{B_{0}} .
$$

Combining (25) and (30), we can conclude that the operator $T$ is contraction.

Step-5: In this step we establish priori bounds.

To complete this step, it is sufficient to prove that the set

$$
P=\left\{w \in B_{0}: w=\lambda T(w / \lambda)+\lambda S(w), \text { for some } \lambda \in(0,1)\right\}
$$

is bounded. Let $w \in P$, then we have $w=\lambda T(w / \lambda)+\lambda S(w)$. Thus, for each $(x, y) \in J$, we have 


$$
\begin{aligned}
& w(x, y) \\
& =\lambda \sum_{k=1}^{m}\left(\left\|I_{k} \frac{\left(w\left(x_{k}^{-}, y\right)\right)}{\lambda}\right\|-\left\|I_{k} \frac{\left(w\left(x_{k}^{-}, 0\right)\right)}{\lambda}\right\|\right) \\
& +\frac{\lambda}{\Gamma\left(r_{1}\right) \Gamma\left(r_{2}\right)} \sum_{k=1}^{m} \int_{x_{k-1}}^{x_{k}} \int_{0}^{y}\left(x_{k}-s\right)^{r_{1}-1}(y-t)^{r_{2}-1} f\left(s, t, \bar{w}_{(s, t)}+v_{(s, t)}\right) d t d s \\
& +\frac{\lambda}{\Gamma\left(r_{1}\right) \Gamma\left(r_{2}\right)} \int_{x_{k}}^{x} \int_{0}^{y}(x-s)^{r_{1}-1}(y-t)^{r_{2}-1} f\left(s, t, \bar{w}_{(s, t)}+v_{(s, t)}\right) d t d s .
\end{aligned}
$$

Using the assumptions $\left(A_{2}\right)$ and $\left(A_{3}\right)$ in (31), we have

$$
\begin{aligned}
& \|w(x, y)\| \\
& \leq \sum_{k=1}^{m} \lambda\left(\left\|I_{k} \frac{\left(w\left(x_{k}^{-}, y\right)\right)}{\lambda}\right\|-\left\|I_{k}(0)\right\|+\left\|I_{k} \frac{\left(w\left(x_{k}^{-}, 0\right)\right)}{\lambda}\right\|-\left\|I_{k}(0)\right\|\right) \\
& +2 \lambda \sum_{k=1}^{m}\left\|I_{k}(0)\right\|+\frac{\|p\|_{\infty}}{\Gamma\left(r_{1}\right) \Gamma\left(r_{2}\right)} \sum_{k=1}^{m} \int_{x_{k-1}}^{x_{k}} \int_{0}^{y}(x-s)^{r_{1}-1}(y-t)^{r_{2}-1} \\
& \left\|\bar{w}_{(s, t)}+v_{(s, t)}\right\|_{B} d t d s+\frac{\|q\|_{\infty}}{\Gamma\left(r_{1}\right) \Gamma\left(r_{2}\right)} \sum_{k=1}^{m} \int_{x_{k-1}}^{x_{k}} \int_{0}^{y}(x-s)^{r_{1}-1}(y-t)^{r_{2}-1} d t d s \\
& +\frac{\|p\|_{\infty}}{\Gamma\left(r_{1}\right) \Gamma\left(r_{2}\right)} \sum_{k=1}^{m} \int_{0}^{x} \int_{0}^{y}(x-s)^{r_{1}-1}(y-t)^{r_{2}-1}\left\|\bar{w}_{(s, t)}+v_{(s, t)}\right\|_{B} d t d s \\
& +\frac{\|q\|_{\infty}}{\Gamma\left(r_{1}\right) \Gamma\left(r_{2}\right)} \sum_{k=1}^{m} \int_{0}^{x} \int_{0}^{y}(x-s)^{r_{1}-1}(y-t)^{r_{2}-1} d t d s \\
& \leq l \sum_{k=1}^{m}\left(\|\left(w\left(t_{k}^{-}, x\right)\|+\| w\left(t_{k}^{-}, 0\right) \|\right)+2 I^{*}\right. \\
& +\frac{\|p\|_{\infty}}{\Gamma\left(r_{1}\right) \Gamma\left(r_{2}\right)} \sum_{k=1}^{m} \int_{x_{k-1}}^{x_{k}} \int_{0}^{y}(x-s)^{r_{1}-1}(y-t)^{r_{2}-1}\left\|\bar{w}_{(s, t)}+v_{(s, t)}\right\|_{B} d t d s \\
& +\frac{\|q\|_{\infty}}{\Gamma\left(r_{1}\right) \Gamma\left(r_{2}\right)} \sum_{k=1}^{m} \int_{x_{k-1}}^{x_{k}} \int_{0}^{y}(x-s)^{r_{1}-1}(y-t)^{r_{2}-1} d t d s \\
& +\frac{\|p\|_{\infty}}{\Gamma\left(r_{1}\right) \Gamma\left(r_{2}\right)} \sum_{k=1}^{m} \int_{0}^{x} \int_{0}^{y}(x-s)^{r_{1}-1}(y-t)^{r_{2}-1}\left\|\bar{w}_{(s, t)}+v_{(s, t)}\right\|_{B} d t d s \\
& +\frac{\|q\|_{\infty}}{\Gamma\left(r_{1}\right) \Gamma\left(r_{2}\right)} \sum_{k=1}^{m} \int_{0}^{x} \int_{0}^{y}(x-s)^{r_{1}-1}(y-t)^{r_{2}-1} d t d s,
\end{aligned}
$$

where $I^{*}=\sum_{k=1}^{m}\left\|I_{k}(0)\right\|$ and

$$
\begin{aligned}
& \left\|\bar{w}_{(s, t)}+v_{(s, t)}\right\|_{B} \\
& \leq\left\|\bar{w}_{(s, t)}\right\|_{B}+\left\|v_{(s, t)}\right\|_{B} \\
& \leq K \sup \{w(\bar{s}, \bar{t}):(\bar{s}, \bar{t}) \in[0, s] \times[0, t]\}+K\|\phi(0,0)\|+M\|\phi\|_{B} \\
& =\sigma(s, t)(\text { say }) .
\end{aligned}
$$


Hence, for each $(t, x) \in J$, we have

$$
\begin{aligned}
\|w(x, t)\| & \leq l \sum_{k=1}^{m}\left(\left\|w\left(t_{k}^{-}, x\right)\right\|+\left\|w\left(t_{k}^{-}, 0\right)\right\|\right)+2 I^{*}+\frac{2 a^{r_{1}} b^{r_{2}}\|q\|_{\infty}}{\Gamma\left(r_{1}+1\right) \Gamma\left(r_{2}+1\right)} \\
& +\frac{\|p\|_{\infty}}{\Gamma\left(r_{1}\right) \Gamma\left(r_{2}\right)}\left[\sum_{k=1}^{m} \int_{t_{k-1}}^{t_{k}} \int_{0}^{x}(t-s)^{r_{1}-1}(x-\eta)^{r_{2}-1} \sigma(s, \eta) d \eta d s\right. \\
& \left.+\int_{0}^{t} \int_{0}^{x}(t-s)^{r_{1}-1}(x-\eta)^{r_{2}-1} \sigma(s, \eta) d \eta d s\right] .
\end{aligned}
$$

Using the inequality (32) and the definition of $\sigma$, we have

$$
\begin{aligned}
\sigma(t, x) & \leq M\|\phi\|_{B}+K\|\phi(0,0)\|+l \sum_{k=1}^{m}\left(\left\|w\left(t_{k}^{-}, x\right)\right\|+\left\|w\left(t_{k}^{-}, 0\right)\right\|\right)+2 I^{*} \\
& +\frac{2 a^{r_{1}} b^{r_{2}}\|q\|_{\infty}}{\Gamma\left(r_{1}+1\right) \Gamma\left(r_{2}+1\right)}+\frac{\|p\|_{\infty}}{\Gamma\left(r_{1}\right) \Gamma\left(r_{2}\right)}\left[\sum_{k=1}^{m} \int_{t_{k-1}}^{t_{k}} \int_{0}^{x}(t-s)^{r_{1}-1}\right. \\
& \left.(x-\eta)^{r_{2}-1} \sigma(s, \eta) d \eta d s+\int_{0}^{t} \int_{0}^{x}(t-s)^{r_{1}-1}(x-\eta)^{r_{2}-1} \sigma(s, \eta) d \eta d s\right] .
\end{aligned}
$$

For any $(t, x) \in J$, the Lemma 2.8 implies that there exists $\bar{\delta}=\bar{\delta}\left(r_{1}, r_{2}\right)$ such that

$$
\begin{aligned}
& \sigma(t, x) \\
& \leq\left(M\|\phi\|_{B}+K\|\phi(0,0)\|+l \sum_{k=1}^{m}\left(\left\|w\left(t_{k}^{-}, x\right)\right\|+\left\|w\left(t_{k}^{-}, 0\right)\right\|\right)+2 I^{*}\right. \\
& \left.+\frac{2 a^{r_{1}} b^{r_{2}}\|q\|_{\infty}}{\Gamma\left(r_{1}+1\right) \Gamma\left(r_{2}+1\right)}\right) \times\left(1+\bar{\delta} \frac{\|p\|_{\infty}}{\Gamma\left(r_{1}\right) \Gamma\left(r_{2}\right)} \int_{0}^{t} \int_{0}^{x}(t-s)^{r_{1}-1}(x-\eta)^{r_{2}-1} d \eta d s\right) \\
& \leq\left(M\|\phi\|_{B}+K\|\phi(0,0)\|+l \sum_{k=1}^{m}\left(\left\|w\left(t_{k}^{-}, x\right)\right\|+\left\|w\left(t_{k}^{-}, 0\right)\right\|\right)+2 I^{*}\right. \\
& \left.+\frac{2 a^{r_{1}} b^{r_{2}}\|q\|_{\infty}}{\Gamma\left(r_{1}+1\right) \Gamma\left(r_{2}+1\right)}\right) \times\left(1+\bar{\delta} \frac{a^{r_{1}} b^{r_{2}}\|p\|_{\infty}}{\Gamma\left(r_{1}+1\right) \Gamma\left(r_{2}+1\right)}\right) \\
& =\bar{\tau}(\text { say }) .
\end{aligned}
$$

Hence, for each $(t, x) \in J$, we have

$$
\|w(t, x)\|_{\infty} \leq \sigma(t, x)
$$

This proves that the set $P$ is bounded.

Therefore, by the consequence of Theorem 2.9 (Burton-Kirk fixed point theorem) we deduce that $S+T$ has a fixed point which is a solution of IVP given by (1) to (4). This completes the proof.

\section{Applications}

In this section, we provide an illustrative example to support the analytic proof of our Theorem 3.1.

\section{Example 4.1.}

Consider the following IVP for FOIPHDEs with infinite delay:

$$
\left(D_{z_{k}}^{r} u\right)(x, y)=\frac{e^{-x-y}}{9+e^{x+y}} \cdot \frac{2+|u(x, y)|}{1+|u(x, y)|},(x, y) \in J=\left[0, \frac{1}{2}\right] \times[0,1] \bigcup\left(\frac{1}{2}, 1\right] \times[0,1],
$$




$$
\begin{gathered}
u\left(\frac{1}{2}^{+}, y\right)=u\left(\frac{1}{2}^{-}, y\right)+\frac{\left|u\left(\frac{1}{2}^{-}, y\right)\right|}{\frac{1}{4}+\left|u\left(\frac{1}{2}^{-}, y\right)\right|}, \text { if } y \in[0,1], \\
u(x, y)=x+y^{2}, \text { if }(x, y) \in[-1,1] \times[-2,1] \backslash(0,1] \times(0,1], \\
u(x, 0)=x, x \in[0,1], u(0, y)=y^{2}, y \in[0,1],
\end{gathered}
$$

where $z_{0}=(0,0), z_{1}=\left(\frac{1}{2}, 0\right)$. Let $\sigma \in \mathbf{R}$ and $C_{\sigma}$ be the set of all piece-wise continuous functions $\phi$ : $(-\infty, 0] \times(-\infty, 0] \rightarrow \mathbf{R}^{n}$ for which a $\operatorname{limit}_{\|} \lim _{\|(s, t)\| \rightarrow \infty} e^{\sigma(s+t)} \phi(s, t)$ exists with the norm

$$
\|\phi\|_{C_{\sigma}}=\sup _{(s, t) \in(-\infty, 0] \times(-\infty, 0]} e^{\sigma(s+t)}\|\phi(s, t)\| .
$$

Set

$$
f(x, y, \varphi)=\frac{e^{-x-y}(2+|\varphi|)}{\left(9+e^{x+y}\right)(1+|\varphi|)},(x, y) \in[0,1] \times[0,1], \varphi \in C
$$

and

$$
I_{1}(u)=\frac{|u|}{\frac{1}{4}+|u|}, u \in \mathbf{R} .
$$

Then, it is clear that the functions $f$ and $I_{1}$ are continuous, and for $(x, y) \in[0,1] \times[0,1]$ and $\varphi \in C$, we have

$$
|f(x, y, \varphi)| \leq \frac{e^{-x-y}}{\left(9+e^{x+y}\right)}(2+|\varphi|) .
$$

Hence assumption $\left(A_{2}\right)$ is satisfied with

$$
p(x, y)=\frac{2 e^{-x-y}}{\left(9+e^{x+y}\right)} \text { and } q(x, y)=\frac{e^{-x-y}}{\left(9+e^{x+y}\right)} .
$$

Now, for $u_{1}, u_{2} \in \mathbf{R}$, we obtain

$$
\left|I_{1}\left(u_{1}\right)-I_{2}\left(u_{2}\right)\right|=\left|\frac{\left|u_{1}\right|}{\frac{1}{4}+\left|u_{1}\right|}-\frac{\left|u_{2}\right|}{\frac{1}{4}+\left|u_{2}\right|}\right| \leq \frac{1}{4}\left|u_{1}-u_{2}\right| .
$$

Thus assumption $\left(A_{3}\right)$ is satisfied with $l=\frac{1}{4}$. Since $l=\frac{1}{4}$, then the condition (25) is also satisfied. That is all the assumptions of Theorem 3.1 are satisfied. Therefore, the Theorem 3.1 implies that the IVP given by (34) to (37) has at least one solution on $(-\infty, 1] \times(-\infty, 1]$.

\section{Conclusion}

In this article, we have proven a new existence criterion for solutions to the initial value problem for FOIPHDEs given by (1) to (4), applying Burton-Kirk fixed point theorem. Using our Theorem 3.1, one can easily be checked the existence of at least one solution of initial value problem given by (1) to (4). The established result provides an easy and straightforward technique to cheek the existence of solutions to the initial value problem for FOIPHDEs given by (1) to (4). Furthermore, the results of this research extend the corresponding results of Abbas et al. [19], Benchohra and Boutefal [27], and Abbas and Benchohra [28, 29, 30, 31].

\section{Competing Interests}

The authors declare that they have no any competing interests. 


\section{Authors Contributions}

All authors read and approved the final version of the manuscript.

\section{Acknowledgment}

We would like to thank the honourable reviewers for their valuable comments which help us to enriched the quality of this article. We are also thankful to the journal authority for their unconditional support.

\section{References}

[1] S. Das, Functional Fractional Calculus, Springer-Verlag, Berlin, Heidelberg (2011).

[2] K. Diethelm, The Analysis of Fractional Differential Equations, Springer (2010).

[3] M.D. Ortigueira, Fractional Calculus for Scientists and Engineers. Lecture Notes in Electrical Engineering, 84. Springer, Dordrecht (2011).

[4] V.E. Tarasov, Fractional Dynamics: Application of Fractional Calculus to Dynamics of Particles, Fields and Media, Springer, Heidelberg; Higher Education Press, Beijing (2010).

[5] V. Lakshmikantham, S. Leela, and J.V. Devi, Theory of Fractional Dynamic Systems, Cambridge Academic Publishers, Cambridge, UK (2009).

[6] S. Das, Functional Fractional Calculus for System Identification and Controls, Springer-Verlag, Berlin, Heidelberg (2008).

[7] J. Sabatier, O.P. Agrawal, and J.A.T. Machado, Eds., Advances in Fractional Calculus: Theoretical Developments and Applications in Physics and Engineering, Springer, Dordrecht, The Netherlands (2007).

[8] A.A. Kilbas, H.M. Srivastava, and J.J. Trujillo, Theory and Applications of Fractional Differential Equations, vol. 204 of North-Holland Mathematics Studies, Elsevier Science B.V., Amsterdam, The Netherlands (2006).

[9] R. Hilfer, Applications of Fractional Calculus in Physics, World Scientific, Singapore (2000).

[10] I. Podlubny, Fractional Differential Equations, Math. Sci. Eng., Academic Press, San Diego, New York, 1999.

[11] S. Abbas, M. Benchohra and G.M. NGuerekata, Topics in Fractional Differential Equations, Springer, New York (2012).

[12] R. Agarwal, M.P. Yadav, D. Baleanu and S.D. Purohit, Existence and uniqueness of miscible flow equation through porous media with a non-singular fractional derivative, AIMS Mathematics, 5(2) (2020),1062-1073.

[13] M. Asaduzzaman and M Zulfikar Ali, Existence of positive solution to the boundary value problems for coupled system of nonlinear fractional differential equations, AIMS Mathematics, 4(3) (2019), 880-895.

[14] B. Zhu, B. Han and X. Lin, Existence Results for a Class of Semilinear Fractional Partial Differential Equations with Delay in Banach Spaces, Hindawi, J. of Function Spaces, 2019 (2019), 7 pages.

[15] K. Zhang and Z. Fu, Solutions for a Class of Hadamard Fractional Boundary Value Problems with Sign-Changing Nonlinearity, Hindawi Publishing, J. of Function Spaces, 2019 (2019), 7 pages.

[16] P. Agarwal, Q. Al-Mdallal, Y.J. Cho and S. Jain, Fractional differential equations for the generalized Mittag-Leffler function, Adv. Differ. Equs., 2018:58 (2018).

[17] A.A. Hemeda, Solution of Fractional Partial Differential Equations in Fluid Mechanics by Extension of Some Iterative Method, Hindawi Publishing, Abst. Appl. Anal., 2013 (2013), 9 pages.

[18] S. Abbas, M. Benchohra and A. Cabada, Partial neutral functional integro-differential equations of fractional order with delay, Boundary Value Problems, 2012:128 (2012).

[19] S. Abbas, M. Benchohra and L. Gorniewicz, Fractional Order Impulsive Partial Hyperbolic Differential Inclusions with Variable Times, Discussiones Mathematicae, Diff. Incl. Cont. Optim., 31 (2011), 91-114.

[20] S. Abbas and M. Benchohra, A global uniqueness result for fractional order implicit differential equations, Comment.Math.Univ.Carolin., 53(4) (2012), 605-614.

[21] S. Abbas and M. Benchohra, Partial hyperbolic differential equations with finite delay involving the Caputo fractional derivative, Commun. Math. Anal., 7 (2009), 62-72.

[22] S. Abbas and M. Benchohra, Darboux problem for perturbed partial differential equations of fractional order with finite delay, Nonlinear Anal. Hybrid Syst., 3 (2009), 597-604.

[23] R.P. Agarwal, M. Benchohra and S. Hamani, A survey on existence result for boundary value problems of nonlinear fractional differential equations and inclusions, Acta. Appl. Math., 109(3) (2010), 973-1033.

[24] M. Benchohra, J. Henderson and S.K. Ntouyas, Impulsive Differential Equations and Inclusions, Hindawi Publishing Corporation, New York (2006).

[25] M. Benchohra and B.A. Slimani, Existence and uniqueness of solutions to impulsive fractional differential equations, Electron. J. Differential Equations 2009:10 (2009), 11 pages.

[26] A.N. Vityuk and A.V. Golushkov, Existence of solutions of systems of partial differential equations of fractional order, Nonlinear Oscil., 7(3) (2004), 318-325.

[27] M. Benchohra and Z. Boutefal, Impulsive Differential Equations of Fractional Order with Infinite Delay, J. Frac. Cal. Appl., 4(2) (2013), 209-223. 
[28] S. Abbas and M. Benchohra, Upper and Lower Solutions Method for Darboux Problem for Fractional Order Implicit Impulsive Partial Hyperbolic Differential Equations, Acta Univ. Palacki. Olomuc., Fac. rer. nat., Mathematica, 51(2) (2012), 5-18.

[29] S. Abbas and M. Benchohra, Darboux problem for implicit impulsive partial hyperbolic fractional order differential equations. Elect. J. Differ. Equas., 2011:150 (2011), 14 pages.

[30] S. Abbas and M. Benchohra, Upper and Lower Solutions Method for Partial Hyperbolic Differential Equations with CaputoâĂŹs Fractional Derivative, LIBERTAS MATHEMATICA, XXXI (2011), 103-110.

[31] S. Abbas and M. Benchohra, Darboux problem for partial functional differential equations with infinite delay and CaputoâĂŹs fractional derivative. Adv. Dyn. Syst. Appl., 5(1) (2010), 1-19.

[32] J.K. Hale and S.V. Lunel, Introduction to Functional -Differential Equations, Applied Mathematical Sciences, 99, SpringerVerlag, New York (1993).

[33] Y. Hino, S. Murakami and T. Naito, Functional Differential Equations with Infinite Delay, in: Lecture Notes in Mathematics, 1473, Springer-Verlag, Berlin (1991).

[34] V. Kolmanovskii, and A. Myshkis, Introduction to the Theory and Applications of Functional-Differential Equations, Kluwer Academic Publishers, Dordrecht (1999).

[35] V. Lakshmikantham, L. Wen and B. Zhang, Theory of Differential Equations with Unbounded Delay, Mathematics and its Applications, Kluwer Academic Publishers, Dordrecht (1994).

[36] H. Smith, An Introduction to Delay Differential Equations with Applications to the Life Sciences, Springer (2011).

[37] J. Wu, Theory and Applications of Partial Functional Differential Equations, Springer-Verlag, New York (1996).

[38] J. Hale and J. Kato, Phase space for retarded equations with infinite delay, Funkcial. Ekvac. 21, (1978), 11-41.

[39] T. Czlapinski, On the Darboux problem for partial differential-functional equations with infinite delay at derivatives. Nonlinear Anal., 44 (2001), 389-398.

[40] V. Lakshmikantham, D.D. Bainov and P.S. Simeonov, Theory of Impulsive Differential Equations, World Scientific, Singapore (1989).

[41] T.A. Burton and C. Kirk, A fixed point theorem of Krasnoselskii-Schaefer type, Math. Nachr., 189 (1998), $23-31$.

[42] A. Granas and J. Dugundji, Fixed Point Theory, Springer-Verlag, New York (2003).

[43] D. Henry, Geometric theory of Semilinear Parabolic Partial Differential Equations, Springer-Verlag, Berlin-New York (1989).

[44] E. Hernandez, A. Anguraj and M.M. Arjunan, Existence results for an impulsive second order differential equation with state-dependent delay, Dyn. Contin. Discrete Impuls. Syst. Ser. Appl. Math. Anal., 17 (2010), 287-301.

[45] M. FrÂl'chet, Sur quelques points du calculfonctionnel, Rend. Circ. Mat. Palermo, 22 (1906), 1-74. 